Running Head: AMP: HOT OR NOT?

The Affect Misattribution Procedure: Hot or Not?

Christophe Blaison ${ }^{1}$, Roland Imhoff ${ }^{2}$, Isabell Hühnel ${ }^{1}$, Ursula Hess ${ }^{1}$, and Rainer Banse ${ }^{2}$

${ }^{1}$ Humboldt-Universität zu Berlin, Berlin, Germany

${ }^{2}$ University of Bonn, Bonn, Germany

October 2011

Blaison, C., Imhoff, R., Hühnel, I., Hess, U., \& Banse, R. (2012). The Affect Misattribution Procedure: Hot or Not? Emotion, 12, 403-412.

This accepted version of the manuscript may slightly differ from the edited version on the editor's website.

Please address correspondence concerning this article to:

Christophe Blaison

Humboldt-Universität zu Berlin

Math. Nat. Fakultät II, Organisations- und Sozialpsychologie

Rudower Chaussee 18, 12489 Berlin

Phone +49-30-2093-9346

Fax +49-30-2093-9332

E-mail: blaisoch@staff.hu-berlin.de 
Acknowledgment: We are grateful to Ottmar Lipp for advice concerning the fear conditioning procedure, to Eric Moody for providing us with mood induction material, and to Bruno Dauvier and Mario Gollwitzer for advice concerning the statistical analyses.

\begin{abstract}
The Affect Misattribution Procedure (AMP; Payne et al., 2005) is an important tool in implicit social cognition research, but its underlying mechanisms are still unknown. This paper investigates whether, as the name implies, affect-based processes really underlie the AMP. We used a modified AMP that enabled us to separate the influence of affective and non-affective processes. In three studies, evidence for the implication of non-affective processes was consistently found. In contrast, there was no evidence for affect-based processes. Thus, the AMP rather seems cold than hot. The generalizability of the results obtained with the modified AMP is discussed.
\end{abstract}

Word count: 98

Keywords: Implicit measures, AMP, Affect priming, Affect misattribution, Semantic priming, Validity. 
The Affect Misattribution Procedure: Hot or Not?

Implicit social cognition research is popular at least in part due to innovative implicit measures. The Affect Misattribution Procedure (AMP; Payne, Cheng, Govorun, \& Stewart, 2005) has become one important player in this regard. Payne et al.'s paper has been cited over 170 times in the six years since its publication (Nosek, Hawkins, \& Frazier, 2011). The AMP is an affective priming paradigm, but unlike variants of affective priming based on response interference (e.g., Fazio, Sanbonmatsu, Powell, \& Kardes, 1986; Murphy \& Zajonc, 1993), it produces strong effects (average $d=1.25$, Payne et al., 2005), and has good internal consistency (.69 < $\alpha<.90$; Payne et al., 2005; Payne, Burkley, \& Stokes, 2008). Only the Implicit Association Test (IAT; Greenwald, McGhee, \& Schwartz, 1998) has a comparable level of internal consistency among all currently used indirect measures. The AMP has thus quickly become popular among social cognition researchers interested in using a second, conceptually different but reliable, measure of implicit social cognitions. Very little is however known about the AMP's underlying mechanisms. This is a crucial problem because validation and knowledge about possible range of application of any indirect measure requires collecting evidence about the relation between construct and outcome measure (De Houwer, Teige-Mocigemba, Spruyt, \& Moors, 2009; Nosek et al., 2011). The aim of the current paper is to address the nature of the mental process underlying the AMP measure.

\section{The AMP}

The AMP uses a priming procedure inspired by Murphy and Zajonc (1993). In the original version (Payne et al., 2005), the procedure consists of briefly presented primes with positive and negative valence, which are followed by briefly presented, affectively neutral Chinese pictographs. Participants have to judge the visual pleasantness of the pictographs. It is assumed that the affective reaction toward the prime influences the responses without the participants' awareness. If the prime elicits positive affect, then participants make more 
pleasant than unpleasant evaluations of the following pictograph, and vice versa. The proportion of pleasant vs. unpleasant judgments towards the pictographs serves as a proxy for the implicit attitude held toward the primes. For example, participants who had positive opinions of American political candidate John Kerry were more likely to make pleasant ratings of pictographs following a picture of Kerry than of pictographs following a picture of political opponent George W. Bush (Payne et al., 2005; Experiment 5).

In support of the procedure's construct validity and implicitness, a racial attitude AMP correlated above $r=.36$ with self-report attitudes toward Blacks as compared to Whites (Payne et al., 2005), and motivation to control prejudiced responses moderated the relationship. (For similar results see Gawronski, Peters, Brochu, \& Strack, 2008; Imhoff \& Banse, 2009; Payne, Burkley, \& Stokes, 2008; Payne, Govorun, \& Arbuckle, 2008.) There also exists strong evidence for the measure's predictive validity as it predicts judgment and behavioral outcomes above and beyond self-report measures (Payne et al., 2008; Payne, Krosnick, Pasek, Lelkes, Akhtar, \& Tompson, 2009; Payne, McClernon, \& Dobbins, 2007).

\section{The Problem}

The mechanisms underlying the AMP effect remain unclear although affect misattribution is commonly believed to mediate the effect of the primes on the judgments of the pictographs in attitude AMPs (Payne et al., 2005). This view is based on the affect-asinformation research tradition (Schwarz \& Clore, 1996, 2003; Winkelman, Zajonc, \& Schwarz, 1997) which states that individuals faced with an ambiguous stimulus use their current mood as information to answer the question, "How do I feel about this?" This strategy can lead to mistakes. Indeed, the immediacy (Clore, Gasper, \& Garvin, 2001) and aboutness (Higgins, 1998) principles cause people to believe that one's subjective experience is caused by whatever one is focusing on at the moment, yet this clearly is not always the case. The argument with regard to the AMP is that, when trying to make sense of the pictographs, 
participants mistake their affective response to the primes as caused by the Chinese pictographs. The "internal argument" would then be "I feel good, thus the Chinese pictograph must be visually pleasant!"

There is emerging empirical evidence compatible with the claim that the AMP effect is based on affective misattribution. Recently, Oikawa, Aarts, and Oikawa (2011) provided results showing that the AMP effect disappeared when participants had to rate the pleasantness of the prime before rating the pleasantness of the pictograph. According to the authors, this constitutes empirical support for the misattribution account as the affect became bound to the prime and therefore could not be (mis)attributed to the pictograph anymore. Payne, Hall, Cameron and Bishara (2010) provided similar evidence with multinomial tree modeling of an attitude AMP. Manipulating the duration of the pictograph presentation affected the estimation of a misattribution parameter $\mathrm{M}$ of the model. The misattribution rate M was higher when the pictographs were presented quickly than when presented slowly. Payne et al. (2010) attributed this result to the ease participants had to distinguish between their affective reactions toward the prime vs. the pictograph when the pictograph was presented slowly, thus preventing any affect misattribution.

Alongside the "affective" AMP variants like the standard attitude AMP, "semantic" AMP variants have appeared recently. Here, affect misattribution should not play a determinant role. For example, Imhoff, Schmidt, Bernhardt, Dierksmeier, and Banse (2011) designed a sexual preference AMP. Heterosexual and homosexual men and women were asked to guess the meaning of briefly presented Chinese ideographs as "sexual" or "not sexual." The frequency of "sexual" responses increased after priming with pictures of individuals of the preferred sex and increasing sexual maturity. In another adaptation, Deutsch and Gawronski (2009; Experiment 4) asked participants whether target Chinese ideographs following prime words representing either animate or inanimate objects signified 
animate or inanimate objects. Participants' responses were consistent with the semantic meaning of the primes: "animate" decisions were significantly more likely after animate primes than after inanimate primes and vice versa.

What mechanisms could possibly account for these semantic AMP variants? Basing ourselves on Loersch and Payne (2011), we suggest that semantic misattribution takes place. For Loersch and Payne (2011) the effect of primes on higher-order cognitive processes (e.g., judgments) may be indirect. In a first step, priming increases the accessibility of semantically, experientially, or evaluatively related mental content. In a second step, this highly accessible mental content is misattributed to whatever is in the focus of attention to produce distinct effects on judgment, behavior, and motivation. As such, the general principles governing affect misattribution take place also in semantic misattribution (Loersch \& Payne, 2011). For example, when judging whether a pictograph depicts something animated (Deutsch \& Gawronski, 2009), participants would mistake their accessible mental content as caused by the Chinese pictograph when in fact it was caused by the prime: "Pictures of running basset hounds keep popping into my mind, thus the Chinese pictograph may represent something animated!"

Semantic misattribution could also underlie affective AMP variants. Indeed, affect might generally reinforce the activation of congruent affective or emotional semantic information in working memory (i.e., valence attributes: Greenwald, Rudman, Nosek, Banaji, Farnham, \& Mellott, 2002; implicit affect: Quirin, Kazén, \& Kuhl, 2009; emotion concepts: Innes-Ker \& Niedenthal, 2002; see also Bowers, 1981; Forgas, 1999; Storbeck \& Clore, 2007) as when your puppy makes you feel good and activates affectively congruent cognitive representations. Thus, primes in an affective AMP variant could give raise both to affect and congruent cognitive representations so that affective and semantic misattribution processes would run parallel. Alternatively, primes in an affective AMP variant could admittedly give 
raise both to affect and congruent cognitive representations but only semantic or affect misattribution processes would occur in isolation. Finally, affective or emotional cognitive representations could get activated in the absence of any affect. This kind of cognitive priming without affective experience takes place for example when a sentence describing a puppy activates positive cognitive representations without arousing your feelings (e.g., InnesKer \& Niedenthal, 2002; Niedenthal, Rohmann, \& Dalle, 2003). Here, the primes would activate congruent cognitive representation without arousing feelings so that, as there is no affect to misattribute, only semantic misattribution could occur.

In sum, then, only semantic misattribution can account for semantic AMP variants, whereas affect misattribution and semantic misattribution might both account for affective AMP variants. In what follows, we will provide evidence that affect misattribution is less likely to occur than semantic misattribution in an affective AMP variant, and thus all the more perhaps in the AMP in general. In other words, we would like to provide some support for the notion that non-affective or cold processes rather than affect-based or hot processes underlie the AMP.

\section{Process Dissociation}

We have suggested that both affect and semantic misattribution could underlie affective AMP variants. Yet, these two kinds of processes are usually confounded. For example, in attitude AMPs, both affect and semantic misattribution predict that valenced primes lead to congruent judgments of the pictograph: the semantic content of a bunch of puppies is "positive"; the potentially accompanying affective experience also. To create meaningful process dissociation requires a prime with semantic content incongruent with the affective experience it produces. In this regard, angry faces are very good candidates. Indeed, the semantic content of an angry face is "anger" but it is likely to elicit fear. In such case, 
pictographs following angry faces should be judged as related to anger if semantic misattribution takes place, but as related to fear if affect misattribution takes place.

\section{Study 1}

Angry faces have been shown to activate the brain's fear network in highly socially anxious individuals. For example, compared to individuals low in social anxiety, individuals high in social anxiety show an increase in amygdala activity when presented with threatening faces (Phan, Fitzgerald, Nathan, \& Tancer, 2006; Stein, Goldin, Sareen, Zorrilla, \& Brown, 2002; Straube, Kolassa, Glauer, Mentzel, \& Miltner, 2004; Straube, Mentzel \& Miltner, 2005). For purposes of the present study, it is particularly interesting that the amygdala activity difference between high and low socially anxious individuals is especially apparent when participants do not pay explicit attention to the threatening faces (Straube et al., 2004). We took advantage of the individual differences between high and low socially anxious participants in presenting them with a modified AMP featuring angry-face, fearful-face and neutral primes.

If affect misattribution underlies the AMP effect, we would expect that highly socially anxious individuals would be more likely than less socially anxious individuals to associate pictographs following angry-face primes with fear. This expectation is based on the idea that individuals with high levels of social anxiety are indeed afraid when faced with the angryface prime. By contrast, if semantic misattribution underlies the AMP effect, this fear reaction is of no relevance and both groups should associate pictographs following angry-face primes with anger. In addition, the semantic misattribution account predicts more anger responses to pictographs following anger primes than to pictographs following neutral and fear primes, and more anger responses to pictographs following neutral primes than to pictographs following fear primes. Finally, we were interested in the strategies participants used to ascribe meaning to the pictographs; we examined self-reports of strategies to assess 
whether participants responded directly to the prime rather than to the ideographs as such a response strategy would be an alternative explanation of a semantic misattribution effect.

\section{Method}

Participants. A total of 151 German undergraduates (42 men) with a mean age of 27 years $(S D=8)$ from the University of Bonn participated in the study in exchange of course credits.

Material. We selected the five angry and five fearful male faces from the Karolinska Directed Emotional Faces photo set (KDEF; Lundqvist, Flykt, \& Öhman, 1998). They best represented fear and anger according to a normative study by Goeleven, de Raedt, Leyman, and Vershuere (2008). The faces were cropped to eliminate any interfering features such as hairstyle (Goeleven et al., 2008). The pictures were presented in full frontal view with the gaze directed toward the participant. In addition to the angry-face and fearful-face primes, a grey square was used as a neutral control prime. Ninety different Chinese ideographs and the noise-pattern (i.e., a rectangular picture with random shades of grey used to mask the ideographs) were taken from the original set used by Payne et al. (2005).

Instruments. We used a modified AMP. Participants had to decide if a given ideograph either "visually evokes fear" by hitting the left response key or if it "visually evokes anger" by hitting the right response key. For the sake of credibility, we informed participants that, "We know from previous research that the visual aspect of Chinese ideographs induce subtle feelings in observers. This is why we are interested in whether you have the feeling that the visual aspect of the ideographs evokes fear or rather anger." Each trial began with the presentation of the prime (angry face, fearful face, or neutral grey square) for $75 \mathrm{~ms}$ followed a blank screen for $125 \mathrm{~ms}$. The Chinese pictograph appeared for $200 \mathrm{~ms}$ and was backwards masked with the noise-pattern until the participant responded. Labels remained on the screen throughout the task to remind the participants to press the left key if 
the target pictograph visually evoked fear and the right key if it visually evoked anger. Participants performed six blocks of 15 trials each (for a total of 30 fear trials, 30 anger trials, and 30 neutral trials). Within each block, primes and targets appeared in a fully randomized order without replacement. A difference score (number of "anger-evoking” responses to anger trials - number of "anger-evoking” responses to fear trials) was computed for each block of trials in order to estimate the internal consistency of the modified AMP $(\alpha=0.80)$.

After completing the AMP, participants were asked about their strategies when responding to the AMP ("Please describe briefly how you solved the previous task. We are interested in any thoughts about how you came to a decision regarding the pictographs").

Participants' level of social anxiety was assessed using the German version (Sosic, Gieler, \& Stangier, 2008) of the Social Phobia Inventory (SPIN; Connor, Davidson, Churchill, Sherwood, Foa, \& Weisler, 2000). The SPIN features the subscales fear, avoidance, and physiological symptoms. For each of 17 statements, participants rated on a Likert scale ranging from 0 (Not at all) to 4 (Extremely) the degree to which the statement had applied to them in the last few weeks (e.g., "I'm afraid of people in an authority position", "I would do anything to avoid being criticized"). The internal consistency of the SPIN was $\alpha=0.87$.

Procedure. The experiment took place in individual sessions. Participants first completed the modified AMP, then the SPIN questionnaire, then they gave information about the strategies they used.

\section{Results}

One participant reported knowing the actual meaning of most ideographs and was thus excluded, resulting in a total sample of $N=150$. The affect misattribution account predicts a significant interaction effect between kind of prime (angry face, fearful face, or neutral grey square) and level of anxiety on the proportion of "anger-evoking" responses, and the slope of 
the regression line should be negative for anger trials. By contrast, the semantic misattribution account predicts a main effect for kind of prime such that the proportion of "anger-evoking" responses is highest for anger trials, medium for neutral trials, and lowest for fear trials. To test these predictions while avoiding the use of a median split design with the accompanying loss of power (Aiken \& West, 1991), we conducted a mixed linear model (i.e., multilevel) analysis on the frequency of "aggressive" responses to the pictographs. For this the repeated measures factor Prime (angry face, fearful face, or neutral grey square) was nested within participants. Participants' sex and Prime were fixed factors, Subject was defined as a random factor, and the centered SPIN scores served as a covariate. Results showed a significant main effect of Prime, $F(2,317)=17.64, p<.001$. In line with the semantic misattribution account, individual estimates of the effect of Prime showed that overall, participants gave significantly more "anger-evoking" responses when primed with angry-face primes $(M=.63, S D=.17)$ than when primed with neutral primes $(M=.58, S D=$ $.15)$, estimate $=.05, S E=.02, t(286)=2.24, p=.03$, or fearful-face primes $(M=.49, S D=$ $.18)$, estimate $=-.14, S E=.02, t(289)=5.66, p<.001$. Participants also gave significantly more "fear-evoking" responses when primed with fearful-face primes than when primed with neutral primes, estimate $=-.09, S E=.02, t(278)=3.90, p<.001($ see Figure 1$)$. No other significant main or interaction effect emerged (all $F \mathrm{~s}<1.02$, all $p \mathrm{~s}>.36)^{1}$.

Two independent judges rated participants' response strategies according to four given alternative categories, $V=.76, p<.001$. When the two raters disagreed $(13.2 \%$ of the cases), a third judge made the decision. The most common answer type (50\%) named visual features of the ideographs that were classified as anger- or fear-evoking (e.g., spiky signs as "anger-evoking"). A proportion of $21.3 \%$ of the participants reported that they found the task difficult because the signs did not evoke either fear or anger in them. Only $14 \%$ reported they based their responses on feelings, intuition, or spontaneous reactions. A proportion of $7.3 \%$ 
made explicit reference to the picture primes presented before, sometimes mentioning that they were unable to ignore the emotion shown in the faces. The remaining $7.3 \%$ declined to give any indication of their response strategy. Excluding all participants who explicitly mentioned the influence of the primes did not change the pattern of results, as the only significant effect was a main effect of Prime, $F(2,266)=4.07, p<.01$. Lack of statistical power due to the small number of participants who reported that they based their responses on feelings or intuition prevented us from testing whether the affect misattribution effect was stronger among them.

\section{Discussion}

The results strongly support the semantic misattribution account. Participants gave significantly more "anger-evoking" responses when primed with an angry face than when primed with a neutral stimulus or a fearful face. The affect misattribution account was not supported, although we controlled for judgments based directly on the primes rather than the pictographs.

Nevertheless, it may be argued that this first study conducted a somewhat unfair test of the two accounts because the semantic misattribution account (postulating a main effect) is tested with more statistical power than the affect misattribution account (postulating interaction effects). More generally, because Study 1 relied on an interaction with an individual difference measure, its design is open to response idiosyncrasies from socially anxious participants in comparison with other participants. Hence in Study 2 we used an experimental approach that does not rely on individual differences and we predicted a main effect for semantic as well as for affect misattribution.

\section{Study 2}

Recent evidence suggests that not only socially anxious individuals, but anyone perceives angry faces as more threatening while experiencing a state of diffuse anxiety 
(Moody, McIntosh, Mann, \& Weisser, 2007). Hence, by inducing fear it is possible to increase the threat perceived in angry faces experimentally. If the affect misattribution account holds true, participants in an anxious state should make more fear-evoking judgments about the pictographs following angry-face primes. If the semantic misattribution account holds true, however, then irrespective of the anxiety manipulation, pictographs following angry faces should be rated as more anger-evoking.

\section{Method}

Participants. Eighty-six French undergraduates (13 men) with a mean age of 21 years $(S D=7)$ from the University of Aix-Marseille participated voluntarily. None of the participants were familiar with either the Chinese language or the AMP.

Materials. The modified AMP material was the same as in Study 1. Two five-minute video clips from the study of Moody et al. (2007) were used for the fear-induction procedure. The fear-evoking clip showed frightened individuals in various situations (extracts from the movies "Silence of the Lambs" and from "The Shining"). The control clip showed a statue in a public garden with several people seated on the ground next to it reading and talking, with an individual occasionally walking past the field of view. There is evidence that this technique provides a strong and specific induction of fear lasting long enough for our purposes (Moody et al., 2007; Weisser, Moody, \& McIntosh, 2004).

Instruments. We used the same modified AMP as in Study $1 ; \alpha=0.75$ for Study 2. As a manipulation check, participants were asked to rate to what degree they had felt happiness, fear, anger, surprise, sadness, and disgust during the video clip using seven-point Likert scales ranging from $1=$ Not at all to $7=$ Extremely (see Moody et al., 2007). Participants took also the state version of the French translation of the State Trait Anxiety Inventory (STAI; Bruchon-Schweitzer \& Paulhan, 1993). 
Procedure. The experiment took place in individual sessions with laptop computers. Participants were randomly assigned to either the experimental $(n=43)$ or the control group $(n=43)$. The experimental group first watched the frightening video clip, then took the modified AMP, then completed the emotion self-report, and finally the STAI. The control group worked through the identical procedure but watched the neutral video clip. The manipulation check was placed after the AMP to avoid interrupting the flow of the experiment (Moody et al., 2007).

\section{Results}

The manipulation check showed that participants in the experimental group reported more fear $(M=4.2, S D=1.9)$ than participants in the control group $(M=1.9, S D=1.4), t(84)=$ $6.5, p<.001, d=1.41$. With the exception of happiness, which decreased significantly in the experimental group $(M=1.4, S D=1.0)$ in comparison to the control group $(M=1.9, S D=$ $1.16), t(84)=2.1, p<.05$, no other differences emerged between the groups. The STAI results mirrored the effect of the manipulation as the experimental group reported significantly more anxiety $(M=2.6, S D=.64)$ than did the control group $(M=1.9, S D=.62)$, $t(84)=5.15, p<.001, d=1.13$.

The proportion of "anger-evoking" responses to the Chinese ideographs was analyzed with a 2 (Emotion induction: fear versus neutral) $\times 3$ (Prime: anger, fear, and neutral) mixedmodel ANOVA. A significant main effect of Prime emerged, $F(2,252)=22.67, p<.001$. Consistent with the semantic misattribution account, post-hoc tests showed that participants gave significantly (or marginally significantly) more "anger-evoking" responses when primed with angry-face primes $(M=.58, S D=.15)$ than when primed with neutral primes $(M=.53$, $S D=.17), p=.06$, or fearful-face primes $(M=.43, S D=.13), p<.001$. Participants gave significantly fewer "anger-evoking" and thus more "fear-evoking" responses when primed with fearful-face primes than when primed with neutral primes, $p<.001$ (Figure 2). No other 
main effects or interactions emerged. Finally, when controlling for baseline responses on neutral trials ${ }^{2}$, no emotion-induction effect emerged for corrected anger trials (experimental group: $M=.06, S D=.18$; control group: $M=.05, S D=.16), t(84)=.26, p=.80$, nor for corrected fear trials (experimental group: $M=-.09, S D=.20$; control group: $M=-.10, S D=-$ $.19), t(84)=.22, p=.83$.

\section{Discussion}

As in Study 1, results of Study 2 showed strong support for semantic misattribution. Participants responded to the pictographs in a prime-consistent way, regardless of the emotion induction. Despite the stronger power of the design, there was no support for the occurrence of affect misattribution. Nevertheless, two general limitations of Study 2 as well as Study 1 should be taken into consideration.

First, one could argue that there was no direct evidence that the angry faces did indeed elicit more of an automatic fear reaction in socially anxious individuals (Study 1) or in individuals in a diffuse state of anxiety (Study 2). We relied upon previously published and established effects instead of including an explicit manipulation check. A second general limitation resides in the fact that participants made a dichotomous categorization in the context of two types of primes that are semantically and affectively related to the categorization response. This may determine how participants represent the task. An obvious way to make sense of the task would be to map the primes onto the dichotomous categorization response. In the present design this would bias responses towards mapping the semantic meaning of the primes rather than the affective reaction to the primes onto the categorization response because the semantic meaning of the primes and the categorization response have a similar distribution: two types of primes, two response options. Mapping the affective reaction to the primes onto the dichotomous categorization response is less straightforward, especially for anxious people, because both anger and fear primes can elicit 
fear. In terms of the affective reaction the primes can elicit, angry and fearful faces thus constitute one category. These concerns were addressed in Study 3.

\section{Study 3}

In Study 3, the fear reaction towards angry-face primes was experimentally manipulated with a differential fear-conditioning procedure. Reinforced stimuli (CS+) were paired with an aversive unconditioned stimulus (UCS; an electroshock), whereas intermixed unreinforced stimuli (CS-) were not combined with the UCS (Lipp, 2006). In consequence, after the removal of the aversive UCS, subsequent CS+ presentations should elicit more physiological arousal than CS- presentations. It is well established that fear-conditioned angry faces provoke more fear than non-conditioned angry faces (e.g., Öhman \& Dimberg, 1978; Esteves, Parra, Dimberg, \& Öhman; 1994). In addition, fear-conditioned angry faces produce persistent fear reactions (e.g., Öhman \& Dimberg, 1978; for review see Dimberg \& Öhman, 1996). Importantly, a fear reaction towards a fearful stimulus reliably produces a change in electrodermal activity (Lipp, 2006). More specifically, a fearful stimulus produces a greater skin conductance response (SCR) than a less threatening stimulus (e.g., Esteves et al., 1994).

Thus, in Study 3, previously conditioned angry faces (referred to as CS+ from here forward) and unreinforced angry faces (referred to as CS- from here forward) were used as primes. We excluded fearful faces primes to address the response mapping problem but kept the neutral grey square prime to test for a semantic misattribution effect (more aggressionrelated responses after angry faces compared to neutral prime). Electrodermal activity was continuously recorded while participants completed the modified AMP. To assess the success of the conditioning procedure, the resistance to extinction of the differential fear reaction towards CS+ in contrast to the CS- angry faces during the taking of the AMP was assessed via SCR. Specifically, as not everyone is sensitive to fear-conditioning procedures, we 
selected participants who showed greater SCRs for $\mathrm{CS}+$ than for $\mathrm{CS}^{-}$, as evidence for successful conditioning. Self-reported affective reactions towards the CS+ and CS- were also collected. Irrespective of whether an angry face was a CS+ or a CS-, pictographs following angry faces should elicit more anger judgments than a neutral prime if semantic misattribution takes place. Yet, pictographs following CS+ angry primes should produce more fear judgments than pictographs following CS- angry primes if affect misattribution takes place.

\section{Method}

Participants. Sixty women (aged 18-51 years, $M=25.3, S D=5.0$ ) from the Humboldt-Universität zu Berlin participated in the experiment. They were paid eight Euros for their participation. All participants were right-handed.

\section{Materials.}

Visual stimuli. Four full frontal male faces with the highest facial anger expression recognition rate (pictures M10AN, M05AN, M11AN, M17AN; Goeleven et al., 2008) were selected from the KDEF pictures set (Lundqvist et al., 1998). The images were in black and white and cropped to eliminate interfering features (Goeleven et al., 2008). Two faces were randomly selected to serve as $\mathrm{CS}+$, and the other two faces served as $\mathrm{CS}-$. To emphasize the difference between $\mathrm{CS}+$ and $\mathrm{CS}-$, a white circle was added to the $\mathrm{CS}+$ to frame the faces and a white square was added to the CS- (see Figure 3). The pairing between kind of geometrical figure and kind of angry face was counterbalanced across participants. A grey square served as neutral control prime. The Chinese pictographs and the noise-pattern were the same as used in Studies 1 and 2. The size of all stimuli was $20 \times 20 \mathrm{~cm}$ on the screen.

Electro-tactile stimulus. The electro-tactile stimulus was delivered with a Grass Technologies SD9k Square Pulse Stimulator via two electrodes attached near the wrist, on the volar surface of the participant's right forearm. The SD9k stimulator is a constant current 
research device with a built-in isolation circuit that puts out the voltage necessary to maintain the set current flow up to a maximum of $100 \mathrm{~V}$. The current was set to a maximum of $10 \mathrm{~mA}$ and output voltage was not referred to ground.

\section{Instruments.}

$\boldsymbol{A M P}$. Participants were told that Chinese pictographs were able to convey feelings even for non-Chinese speakers. With that in mind, they were asked to guess whether the concept behind the pictograph "rather had to do with flight (e.g., rabbit, to flee)" (abbreviated "flight" in the following) or "rather had to do with aggression (e.g., tiger, to attack)" (abbreviated "aggression" in the following) and to press correspondingly the left or right key. Participants were warned about the influence of the primes; they should prevent their judgment to be biased (Payne et al., 2005). They were explicitly requested to report the first judgment that came to their mind. The modified AMP consisted of 72 trials. There were 12 consecutive blocks of six trials each (2 CS+, 2 CS-, 2 neutral trials). The trial order was randomized. Block numbers 1, 5, and 9 were not used to compute the AMP score. Instead, a shock followed the $\mathrm{CS}+$ primes in these blocks to maintain the conditioning effect. Each prime was presented for $1,500 \mathrm{~ms}^{3}$. At prime offset, a pictograph appeared for $100 \mathrm{~ms}$ followed by the noise-pattern mask. The mask remained on the screen for $6,000 \mathrm{~ms}$ to permit SCRs recording. Finally, a question mark and response category labels appeared and participants were allowed to press either the left or right key.

Affective Ratings of Faces. Self-reported affective reactions towards the angry faces were collected before conditioning as well as at the end of the study. Participants rated the extent to which each of the four angry faces elicited different kinds of discrete emotions: happiness, anger, fear, sadness, disgust, and surprise. They answered on a six-point Likert scale ranging from $1=$ Not at all to $6=$ Completely. The faces presentation order was counterbalanced across participants. 
Apparatus. Skin conductance was measured via a Mindware bioamplifier and Mindware $\mathrm{Ag} / \mathrm{AgCl}$ standard electrodes $(0.8 \mathrm{~cm}$ in diameter) filled with isotonic TD-246 Skin Conductance Electrode Paste, a $0.05 \%$ saline solution in neutral base (Venables \& Christie, 1980). The two electrodes were attached to the distal phalanges of the first and second digit of the left (non-dominant for all participants) hand. Respiration was monitored using a Mindware Respiratory belt transducer attached around the chest, below the sternum. The BioNex skin conductance coupler provided constant voltage of $0.5 \mathrm{~V}$ across the electrodes, processing the signal with a resolution of $0.0015 \mu \mathrm{S}$ (Fowles, Christie, \& Edelberg, 1981). Skin conductance and respiration signals were recorded continuously during the whole experiment with a sampling rate of $1,000 \mathrm{~Hz}$.

Procedure. Upon participants' arrival, a female experimenter informed the participants about the procedure including the application of the electric shocks. After giving written informed consent, participants reclined in a comfortable chair and physiological sensors were attached. The experimenter could monitor the experiment via a video camera and communicate with the participant via microphones. The mean temperature of the laboratory was $23.9^{\circ} \mathrm{C}$. The mean humidity was $32.7 \%$.

In the conditioning-habituation phase, each participant was instructed to look repeatedly at the four angry faces and the grey square. The pictures were randomly presented for 1,500 $\mathrm{ms}$ in three different presentation blocks. Intertrial intervals (ITIs) varied between 4,000 ms and 6,000 ms. Participants then reported their affective reactions towards the faces. Following habituation, the experimenter asked whether the participants would consent to continue with the experiment, including the application of electric shocks. After oral consent was given, the shock electrodes were attached and shock intensity was set so that the participant described it as "annoying but not painful." 
In the conditioning-acquisition phase, participants were told they would see multiple series of pictures sometimes followed by electric shocks. The conditioning consisted of 16 acquisition trials in which the participants watched randomly presented series of angry faces, each presented for 6,000 ms. Two of the faces (CS+) were always paired with an electric shock at stimulus offset, whereas the other two faces (CS-) were not. Electric shocks were delivered for $200 \mathrm{~ms}$. ITIs varied between 8,000 ms and 12,000 ms. Following conditioning, participants received the AMP instructions including the information that shocks could still occur from time to time. After the AMP, participants rated again their affective reactions towards the angry faces. Finally, all physiological sensors were removed and participants were carefully debriefed and paid.

Data Preparation. The scores for the two CS+ faces and the two CS- faces were aggregated into mean scores and will be reported simply as CS+ and CS- scores.

Skin Conductance Responses. SCRs in combination with respiration were visually inspected for signal artifacts. Trials with respiratory artifacts were removed from analyses. SCR (in Microsiemens, $\mu \mathrm{S}$ ) was defined as the largest increase in skin conductance between one and four seconds after picture onset. The minimal response criterion was $0.05 \mu \mathrm{S}$ (Dawson, Schell, \& Filion, 2000). SCR magnitude was computed as the mean value across all stimulus presentations including those without a measurable SCR ("zero response"; Dawson el al., 2000). Zero responses were not excluded; the difference in SCRs between CS + and CS- would otherwise be underestimated. The raw SCR scores were log-transformed to normalize the distributions (Venables \& Christie, 1980).

$\boldsymbol{A M P}$. For each kind of prime, the proportion of "aggression" responses (number of "aggression" responses divided by the total number of trials for one given prime) was computed to obtain three AMP-scores within each AMP block. To assess reliability, we computed difference scores between the proportion of "aggression" responses to the CS+ 
trials and to the CS- trials within each AMP block. We then ran a Cronbach alpha on the three resulting scores. The estimated internal consistency of the modified AMP was $\alpha=0.48$.

\section{Results \& Discussion}

Data from seven participants had to be removed from the analysis. Four were familiar with Chinese pictographs, two showed too many skin conductance artifacts, and the debriefing revealed that one participant felt strongly attracted towards one of the facial stimuli. A prerequisite for testing the affect misattribution account is for participants to actually feel fearful when confronted with the CS+ angry faces during the AMP. To provide the maximum power to test the affect misattribution account, only participants who showed a differential conditioned reaction between the $\mathrm{CS}+$ and $\mathrm{CS}-\left(\mathrm{SCR}_{\mathrm{CS}+}-\mathrm{SCR}_{\mathrm{CS}-}>0\right)$ were selected for further statistical analysis $(\mathrm{n}=34)$. For this group, results show that SCRs during the taking of the modified AMP differed significantly across type of prime, $F(1,39)=19.44$, $p<.05$. As expected, $\mathrm{CS}+$ primes $(M=0.17, S D=0.17)$ elicited significant larger SCRs than CS- primes $(M=0.06, S D=0.08), F(1,33)=20.15, p<.01, \mathrm{r}=.62$, while SCRs for the CSprime did not differ significantly from SCRs for the neutral prime $(M=0.05, S D=0.06)$, $F(1,33)=1.98, p=.17$

Analyses of the emotional self-report data showed that at the end of the procedure, the participants reported more fear towards $\mathrm{CS}+$ angry faces $(M=3.63, S D=1.52)$ than towards CS- angry faces $(M=2.46, S D=1.33), t(33)=4.816, p<.01, r=.38$. Participants also reported stronger anger toward $\mathrm{CS}+(M=3.87, S D=1.44)$ than toward $\mathrm{CS}-(M=3.25, S D=$ 1.52). An additional paired $t$-test showed that the difference between the fear and anger ratings differed significantly between $\mathrm{CS}^{-}$and $\mathrm{CS}+, t(33)=-2.26, p<.05, r=.18$. The $\mathrm{CS}-$ fear-anger difference $(M=0.79, S D=1.46)$ was significantly larger than the $\mathrm{CS}+$ fear-anger difference $(M=0.24, S D=1.59)$. Furthermore, self-reported fear toward CS+ correlated positively with SCR intensity in the CS+ trials, $\rho=.43, p=.01$, whereas self-reported anger 
towards the CS+ did not, $\rho=.27, p=.12$. Thus, there is converging evidence that SCR intensity in CS+ trials represented physiological arousal related to fear.

AMP score analyses. AMP scores for each kind of trial were computed as the proportion of aggression responses (i.e., number of aggression responses / number of trials $\times$ 100). If the semantic misattribution account holds, then the proportion of aggressive responses should be higher for both $\mathrm{CS}+$ and $\mathrm{CS}-$ primes than for neutral primes. Alternatively, pictographs following CS + angry primes should elicit more fear judgments than pictographs following CS- angry primes if affect misattribution takes place. A one-way repeated-measures ANOVA with type of prime as a within-subject factor was conducted on the proportion of aggression responses toward the pictographs. Planned contrasts were conducted to compare (1) neutral trials vs. mean CS- and CS+ trials to test the semantic misattribution account, and (2) $\mathrm{CS}-$ trials vs. CS+ trials to test the affect misattribution account. A significant main effect of prime emerged, $F(2,66)=3.71, p<.05, r=.23$ (Figure 4). The proportion of aggression responses to neutral prime trials $(M=.51, S D=.15)$ was significantly smaller than the mean proportion of aggression responses to CS - and CS+ trials $(M=.59, S D=.14), F(1,33)=6.89, p<.05, r=.42$. This supports the semantic misattribution account. No significant difference in the proportion of aggression responses between the $\mathrm{CS}-(M=.57, S D=.16)$ and $\mathrm{CS}+$ trials $(M=.61, S D=.20)$ was found, $F(1,33)$ $=1.24, p=.27$. Since the pictographs preceded by the faces that had been fear-conditioned were not associated with more fear responses than the pictographs preceded by faces that had not been conditioned, these results provide no support for the affect misattribution account.

Study 3 addressed two general concerns that could have potentially affected the results of Studies 1 and 2. Specifically, a concern remained that participants may not have experienced fear when fear was expected. However, the SCRs assessed in Study 3 served as confirmation that CS+ indeed elicited fear. Furthermore, the concern that response mapping 
could explain the results was resolved by presenting only angry-faces and neutral primes. Yet, even after these alternative explanations were controlled for, the results supported the semantic misattribution account but not the affect misattribution account.

\section{General Discussion}

The aim of this research was to examine whether affect misattribution or semantic misattribution drove the effect in an affective AMP variant. Three studies pitted the two accounts against each other using different kind of manipulations. Consistent support for semantic misattribution but no evidence in favor of affect misattribution was found. Whether participants were high in trait social anxiety (Study 1), put in an anxious state (Study 2), or confronted with fear-conditioned stimuli (Study 3), meaning of rather than fearful reactions toward angry-face primes transferred onto subsequent pictographs. As even in an affective AMP variant no trace of affect-based processes was found, our results are consistent with the notion that the AMP in general is rather cold than hot. Is the "A" in AMP thus problematic? Before any conclusion, we should first discuss whether the predominance of cold processes in our affective AMP variant also applies to standard attitude AMPs.

Indeed, attitude AMPs (e.g., Payne et al., 2005) require participants to make rough evaluative judgments of the pictographs whereas the modified AMP required more specific, emotion-related judgments. Given that emotions have a more clearly identifiable referent (Zajonc, 1998), one could argue that it may be more difficult to misattribute fear than unclearly defined positive or negative affective states (Ekman, 1984; Fridja, 1986; Schwarz \& Clore, 1993; Sechrist, Swim, \& Mark, 2003). This would undermine the detection of affect misattribution in the modified AMP in comparison with standard attitude AMPs. As a result, it is perfectly legitimate to wonder whether the findings obtained with our affective AMP variant also hold for standard attitude AMPs. 
A first argument against the lack of affect misattribution in the modified AMP (although it would take place in traditional attitude AMPs) lies in evidence supporting the notion that even when the source of affect is clearly identified, affect can still be misattributed. For example, Keltner, Locke and Audrain (1993) have shown that a negative mood explicitly identified as produced by the features of the room in which participants were seated still influenced their reports of life satisfaction. In theory, thus, felt anger or felt fear could still influence the ratings of the pictographs even if the primes constituted clear referents. Second, in our view there is little reason why participants should be less aware of the source of their affect in an attitude AMP than in the modified AMP. In both, participants are clearly instructed how the primes could influence their judgment. In both, the primes are presented supraliminally. In both, at least when looking at Study 1 and 2, there is a response mapping between features of the prime (e.g., positive vs. negative) and features of the response choice (e.g., pleasant vs. unpleasant). In our opinion, the likelihood of affect misattribution processes is thus as high in the modified AMP as in standard attitude AMPs. Third, it is difficult to explain the lack of affect misattribution in the modified AMP with the semantic misattribution effect consistently showing up. As a general difficulty for misattribution processes to occur in the modified AMP cannot be put forward, one is tempted to conclude that affect misattribution was absent because affect, albeit present, was not misattributed. Of course, the generalization of the results obtained here with the modified AMP to other affective AMP variants like standard attitude AMPs (e.g., Payne et al., 2005) remains open until direct evidence is available. At present, however, our results should invite doubts about whether the "A" of the AMP may be a misnomer.

Another potential limitation resides in the fact that our research was dedicated to the hot vs. the cold nature of the AMP with a focus on affect (hot) vs. semantic (cold) misattribution. However, although our data support the notion of a cold process underlying 
the modified AMP, they do not provide direct evidence for the claim that this cold process is a misattribution process (but see Oikawa et al., 2011; Payne et al., 2010). It is conceivable that the underlying process is not (or not only) an indirect one in which a reaction to the primes is misattributed to the target but a direct one in which the primes directly influence the response or the perception of the pictograph. That is, a semantic category rendered highly accessible would simply bias the behavioral decision toward the congruent response key through the learned association between semantic category and response key. Alternatively, primes could influence the visual processing of the ambiguous pictographs' gestalt so that the visual impression is congruent with information rendered highly accessible by the prime (see for example Balcetis \& Dunning, 2006).

Although our results do not speak to the question of whether indirect misattribution or direct priming or both underlie the AMP, they would suggest that the dominance of cold (vs. hot) processes would also hold for priming processes in the modified AMP. As such, anger information coming from the prime in anger trials would win over the fear information coming from the subjective state of the participant in directly influencing the response decision or the visual processing through spreading of activation. Future research may further try to disentangle priming from misattribution processes in AMP effects and, more generally, it should explore the issue as to why information coming from the prime but not information coming from the subjective affective state of the participant transfers to the target pictographs.

If future research provides further evidence for the cold nature also of attitude AMPs, would it mean that the AMP's wide-spread popularity is unjustified? We would strongly argue against such an interpretation of the results. Quite on the contrary, the important implication of the present work for the future use of the AMP is that it is not restricted to evaluation but could be an "inkblot" for many semantically defined 
psychological constructs; an almost universal, psychometrically sound, projective task. Broadening the applicability of the AMP beyond the evaluative sphere turns this measure into an exciting alternative to the IAT. Of particular interest, results obtained with both measures could be used according to the general principle of convergence: Diagnostic conclusions about individual differences could be drawn with greater confidence if they were based on several conceptually different, convergent, and valid, indirect measures (De Houwer, TeigeMocigemba, Spruyt, \& Moors, 2009; Deutsch \& Gawronski, 2009). Thus, having two different and effective implicit social cognition measures will provide researchers with the generalization power one necessary lacks when results depend on a unique measurement instrument. 


\section{References}

Aiken, L. S., \& West, S. G. (1991). Multiple regression: Testing and interpreting interactions. Newbury Park, CA: Sage.

Balcetis, E., \& Dunning, D. (2006). See what you want to see: Motivational influences on visual perception. Journal of Personality and Social Psychology, 91, 612-625.

Bower, G. H. (1981). Mood and memory. American Psychologist, 36, 129-148.

Bruchon-Schweitzer, M., \& Paulhan, I. (1993). Manuel de l'inventaire d'anxiété état-trait forme $Y(S T A I-Y)$. Paris: ECPA.

Clore, G. L., Gasper, K., \& Garvin, E. (2001). Affect as information. In J. P. Forgas (Ed.), Handbook of affect and social cognition (pp. 121-144). Mahwah, NJ: Lawrence Erlbaum Associates Publishers.

Connor, K. M., Davidson, J. R. T., Churchill, L. E., Sherwood, A., Foa, E., \& Weisler, R. H. (2000). Psychometric properties of the Social Phobia Inventory (SPIN): New selfrating scale. British Journal of Psychiatry, 176, 379-386.

Dawson, M. E., Schell, A. M., \& Filion, D. L., (2000). The electrodermal system. In J. T. Cacioppo, L. G. Tassinary, \& G. G. Bernston (Eds.), Handbook of psychophysiology 2nd ed (pp. 200-223). Boston: Cambridge University Press,

De Houwer, J., Teige-Mocigemba, S., Spruyt, A., \& Moors, A. (2009). Implicit measures: A normative analysis and review. Psychological Bulletin, 135, 347-368

Deutsch, R., \& Gawronski, B. (2009). When the method makes a difference: Antagonistic effects on "automatic evaluations" as a function of task characteristics of the measure. Journal of Experimental Social Psychology, 45, 101-114.

Dimberg, U. (1987). Facial reactions, autonomic activity and experienced emotion: A three component model of emotional conditioning. Biological Psychology, 24, 105-122. 
Dimberg, U., \& Öhman, A. (1996). Behold the wrath: Psychophysiological responses to facial stimuli. Motivation and Emotion, 20, 149-182.

Esteves, F., Parra, C., Dimberg, U., \& Öhman, A. (1994). Nonconscious associative learning: Pavlovian conditioning of skin conductance responses to masked fear-relevant facial stimuli. Psychophysiology, 31, 375-385.

Fazio, R. H., Sanbonmatsu, D. M., Powell, M. C., \& Kardes, F. R. (1986). On the automatic activation of attitudes. Journal of Personality and Social Psychology, 50, 229-238.

Forgas, J.P. (1999). Network theories and beyond. In T. Dalgleish \& M. Power (Eds.) The handbook of cognition and emotion (pp. 591-612). Chichester, UK: Wiley.

Gawronski, B., Peters, K., Brochu, P., \& Strack, F. (2008). Understanding the relations between different forms of racial prejudice: A cognitive consistency perspective. Personality and Social Psychology Bulletin, 34, 648-665.

Fowles, D. C., Christie, M. J., \& Edelberg, R. (1981). Publication recommendations for electrodermal measurements. Psychophysiology, 18, 232-239.

Goeleven, E., De Raedt, R., Leyman, L., \& Verschuere, B. (2008). The Karolinska Directed Emotional Faces: A validation study. Cognition and Emotion, 22, 1094-1018.

Greenwald, A. G., McGhee, D. E., \& Schwartz, J. L. K. (1998). Measuring individual differences in implicit cognition: the implicit association test. Journal of Personality and Social Psychology, 74, 1464-1480.

Greenwald, A. G., Banaji, M. R., Rudman, L. A., Farnham, S. D., Nosek, B. A., \& Mellott, D. S. (2002). A unified theory of implicit attitudes, stereotypes, self-esteem, and selfconcept. Psychological Review, 109, 3-25.

Gross, J. J. (1998). Antecedent- and response-focused emotion regulation: Divergent consequences for experience, expression, and physiology. Journal of Personality and Social Psychology, 74, 224-237. 
Higgins, E. T. (1998). The aboutness principle: A pervasive influence on human inference. Social Cognition, 16, 173-198.

Higgins, E. T., \& Brendl, C. M. (1995). Accessibility and applicability: Some "activation rules" influencing judgment. Journal of Experimental Social Psychology, 31, 218243.

Imhoff, R., \& Banse, R. (2009). Ongoing Victim Suffering Increases Prejudice: The Case of Secondary Anti-Semitism. Psychological Science, 20, 1443 -1447.

Imhoff, R., Schmidt, A. F., Bernhardt, J., Dierksmeier, A., \& Banse, R. (2011). An inkblot for sexual preference: A semantic variant of the Affect Misattribution procedure. Cognition and Emotion, 25, 676-690.

Innes-Ker, Å., \& Niedenthal, P. M. (2002). Emotion concepts and emotional states in social judgment and categorization. Journal of Personality and Social Psychology, 83, 804816.

Lipp, O. V. (2006). Human fear learning: Contemporary procedures and measurement. In M. G. Craske, D. Hermans, \& D. Vansteenwegen (Eds.), Fear and learning (pp. 37-52). Washington: American Psychological Association.

Loersch, C., \& Payne, B. K. (2011). The Situated Inference Model. Perspectives on Psychological Science, 6, $234-252$.

Lundqvist, D., Flykt, A., \& Öhman, A. (1998). The Karolinska Directed Emotional Faces KDEF, CD ROM from Department of Clinical Neuroscience, Psychology section, Karolinska Institutet, ISBN 91-630-7164-9.

Moody, E. J., McIntosh, D. N., Mann, L. J., \& Weisser, K. R. (2007). More than mere mimicry? The influence of emotion on rapid facial reactions to faces. Emotion, 7, 447-457. 
Murphy, S. T., \& Zajonc, R. B. (1993). Affect, Cognition, and Awareness: Affective Priming With Optimal and Suboptimal Stimulus Exposures. Journal of Personality and Social Psychology, 64, 723-739.

Niedenthal, P. M., Rohmann, A., \& Dalle, N. (2003). What is primed by emotion concepts and emotion words? In J. Musch \& K. C. Klauer (Eds.), The psychology of evaluation: Affective processes in cognition and emotion (pp. 307-333). Mahwah, NJ: Lawrence Erlbaum.

Nosek, B. A., Hawkins, C. B., \& Frazier, R. S. (2011). Implicit social cognition: From measures to mechanisms. Trends in Cognitive Sciences, 15, 152-159.

Öhman, A., \& Dimberg, U. (1978). Facial expressions as conditioned stimuli for electrodermal responses: A case of "preparedness?" Journal of Personality and Social Psychology, 36, 1251-1258.

Oikawa, M., Aarts, H., \& Oikawa, H. (2011). There is a fire burning in my heart: The role of causal attribution in affect transfer. Cognition and Emotion, 25, 156-163.

Payne, B. K., Burkley, M. A., \& Stokes, M. B. (2008). Why do implicit and explicit tests diverge? The role of structural fit. Journal of Personality and Social Psychology, 94, $16-31$.

Payne, B. K., Govorun, O., \& Arbuckle, N. L. (2008). Automatic attitudes and alcohol: Does implicit liking predict drinking? Cognition and Emotion, 22, 238-271.

Payne, B. K., Hall, D. L., Cameron, C. D., \& Bishara, A. J. (2010). A process model of affect misattribution. Personality and Social Psychology Bulletin, 36, 1397-1408.

Payne, B. K., McClernon, F., \& Dobbins, I. (2007). Automatic affective responses to smoking cues. Experimental and Clinical Psychopharmacology, 15, 400-409. 
Payne, B. K., Cheng, C. M., Govorun, O., \& Stewart, B. D. (2005). An inkblot for attitudes: Affect misattribution as implicit measurement. Journal of Personality and Social Psychology, 89, 277-293.

Payne, B. K., Krosnick, J. A., Pasek, J., Lelkes, Y., Akhtar, O., \& Tompson, T. (2009). Implicit and explicit prejudice in the 2008 American presidential election. Journal of Experimental Social Psychology, 46, 367-374.

Phan, K. L., Fitzgerald, D. A., Nathan, P. J., \& Tancer, M. E. (2006). Association between amygdala hyperactivity to harsh faces and severity of social anxiety in generalized social phobia. Biological Psychiatry, 59, 424-429.

Quirin, M., Kazén, M., \& Kuhl, J. (2009). When nonsense sounds happy or helpless: The Implicit Positive and Negative Affect Test (IPANAT). Journal of Personality and Social Psychology, 97, 500-516.

Schwarz, N., \& Clore, G. L. (1996). Feelings and phenomenal experiences. In E. T. Higgins \& A. W. Kruglanski (Eds.), Social psychology: Handbook of basic principles (pp. 433-465). New York, NY: Guilford Press.

Schwarz, N., \& Clore, G. L. (2003). Mood as information: 20 years later. Psychological Inquiry, 14, 296-303.

Siddle, D. A. T. (1985). Effects of stimulus omission and stimulus change on dishabituation of the skin conductance response. Journal of Experimental Psychology: Learning, Memory and Cognition, 11, 206-216.

Smith, E. R., \& Neumann, R. (in press). Emotion Considered From the Perspective of DualProcess Models. In L. Feldman-Barrett, P. Niedenthal, \& P. Winkielman (Eds.), Emotion: Conscious and unconscious. New York: Guilford. 
Sosic, Z., Gieler, U., \& Stangier, U. (2008). Screening for social phobia in medical in- and outpatients with the German version of the Social Phobia Inventory (SPIN). Journal of Anxiety Disorders, 22, 849-859.

Stein, M. B., Goldin, P. R., Sareen, J., Zorrilla, L. T. E., \& Brown, G. G. (2002). Increased amygdala activation to angry and contemptuous faces in generalized social phobia. Archive of General Psychiatry, 59, 1027-1034.

Storbeck, J., \& Clore, G. L. (2007). On the interdependence of cognition and emotion. Cognition \& Emotion, 21, 1212-1237.

Strack, F., \& Deutsch, R. (2004). Reflective and impulsive determinants of social behavior. Personality and Social Psychology Review, 8, 220-247.

Straube, T., Mentzel, H.-J., \& Miltner, W. H. R. (2005). Common and distinct brain activation to threat and safety signals in social phobia. Neuropsychobiology, 52, 163168.

Straube, T., Kolassa, I.-T., Glauer, M., Mentzel, H.-J., \& Miltner, W. H. R. (2004). Effect of task conditions on brain responses to threatening faces in social phobics: An eventrelated functional magnetic resonance imaging study. Biological Psychiatry, 56, 921930.

Venables, P. H., \& Christie, M. J. (1980). Electrodermal activity. In I. Martin \& P. H. Venables (Eds.), Techniques in psychophysiology (pp. 3-67). Chichester, UK: Wiley.

Weisser, K. R., Moody, E. J., \& McIntosh, D. (2004, July). Do audio-visual stimuli generate more fear than audio-only stimuli? Poster session presented at the annual meeting of the American Psychological Association, Honolulu, HI.

Winkielman, P., Zajonc, R. B., \& Schwarz, N. (1997). Subliminal affective priming resists attributional interventions. Cognition \& Emotion, 11, 433-465. 
Zajonc, R. B. (1998). Emotions. In D. T. Gilbert, S. T. Fiske \& G. Lindzey (Eds.), The handbook of social psychology, Vols. 1 and 2 (4th ed.) (pp. 591-632). New York: McGraw-Hill. 
AMP: Hot or Not? 34

\section{Footnotes}

${ }^{1}$ To eliminate the possibility that the interaction was not found due to restricted variance in social anxiety we ran additional analyses with clinically relevant social anxiety as a categorical variable. Of the total Study 1 sample, 17 participants were identified as suffering from clinically relevant social anxiety according to the cut-off for the German scale proposed by Sosic et al. (2008). The additional analyses did not show an interaction of prime type and social anxiety, nor did socially anxious participants differ from the rest of the sample on the anger trials. Our results can thus not be explained by reduced variance or too low levels of social anxiety.

${ }^{2}$ In comparison with the control group, the fear induction maybe affected the baseline rate of anger-evoking responses of the experimental group. Independently of kind of prime, participants in a fearful state could have given less anger-evoking responses in general. Thus, in both groups, we computed corrected scores for anger and fear trials in subtracting the mean proportion of "angerevoking" responses to neutral trials from the mean proportion of "anger-evoking" responses to anger and fear trials.

${ }^{3}$ Pretests showed that shorter presentation of stimuli did not produce reliable fearconditioning effects when used in the acquisition phase nor when used in the extinction phase. 


\section{Figure Captions}

Figure 1. Frequency of "anger-evoking" (vs. "fear-evoking") responses (+/- SE) as a function of prime type in Study 1. Error bars show standard errors.

Figure 2. Frequency of "anger-evoking" (vs. "fear-evoking”) responses (+/- SE) as a function of prime type in Study 2. Error bars show standard errors.

Figure 3. Angry-face stimuli used as primes in Study 3.

Figure 4. Frequency of "rather to do with aggression" (vs. "rather to do with flight") responses as a function of prime type in Study 3. 
Figure 1

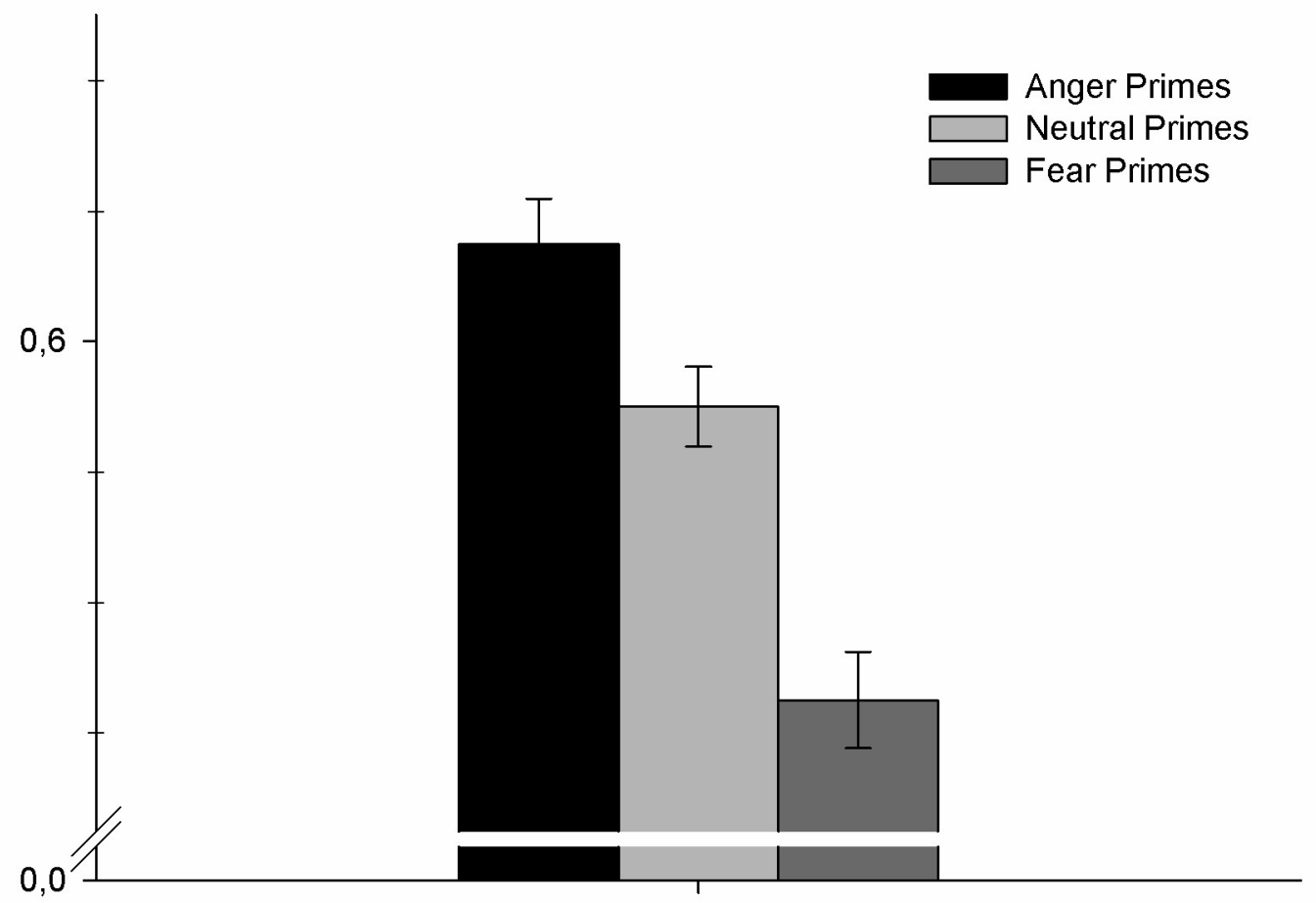

Type of Prime 
Figure 2

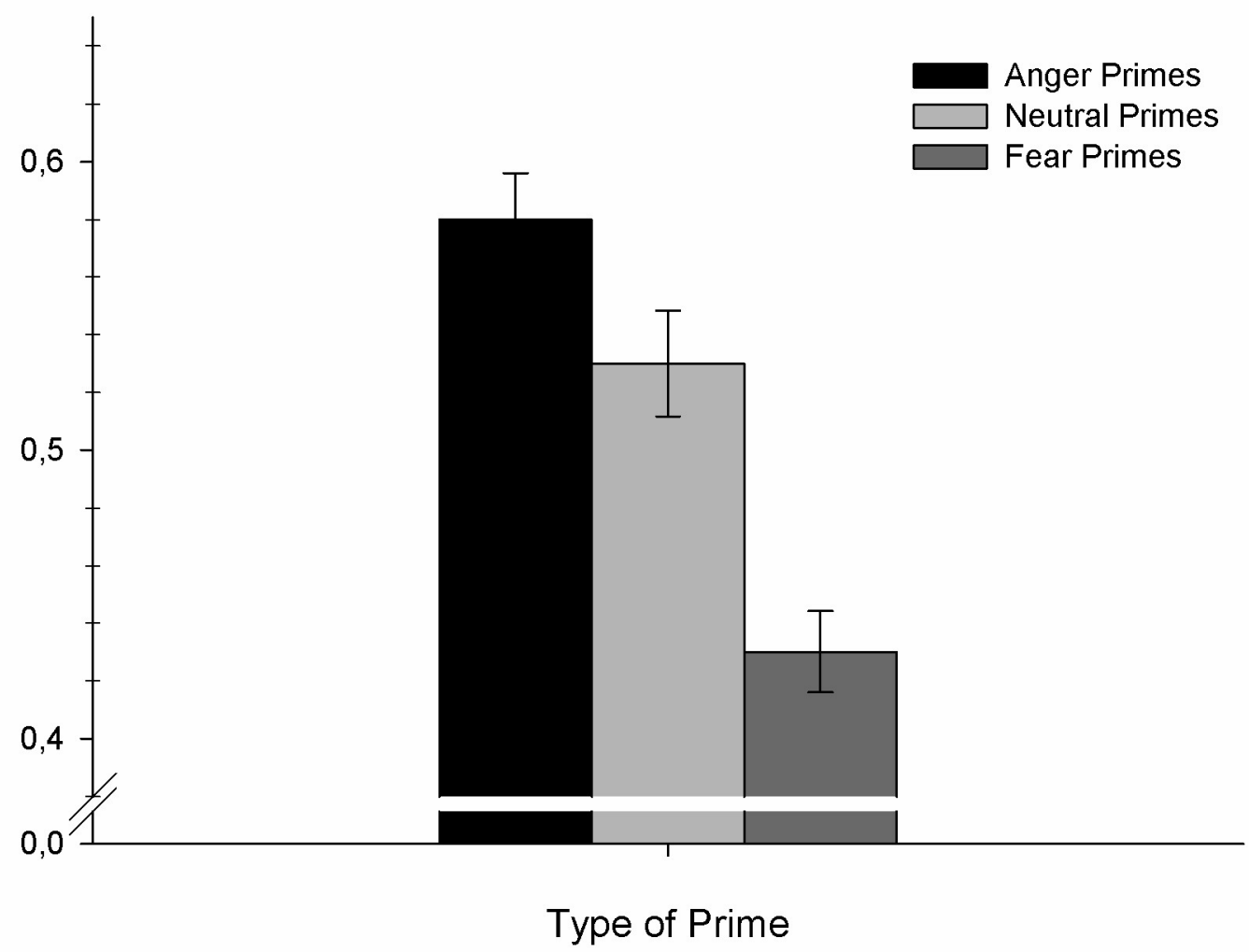


Figure 3
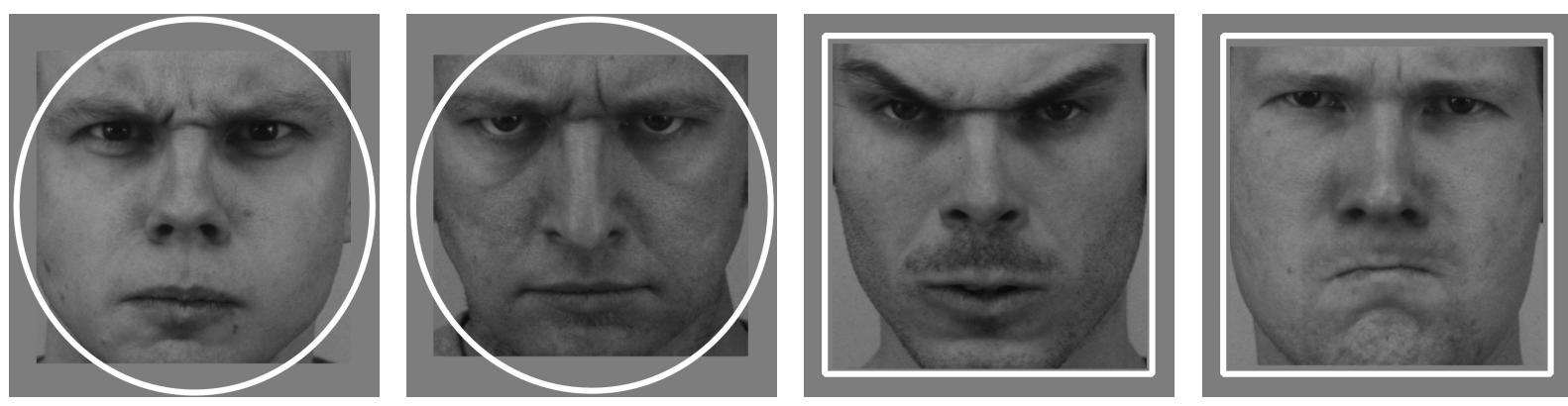
Figure 4

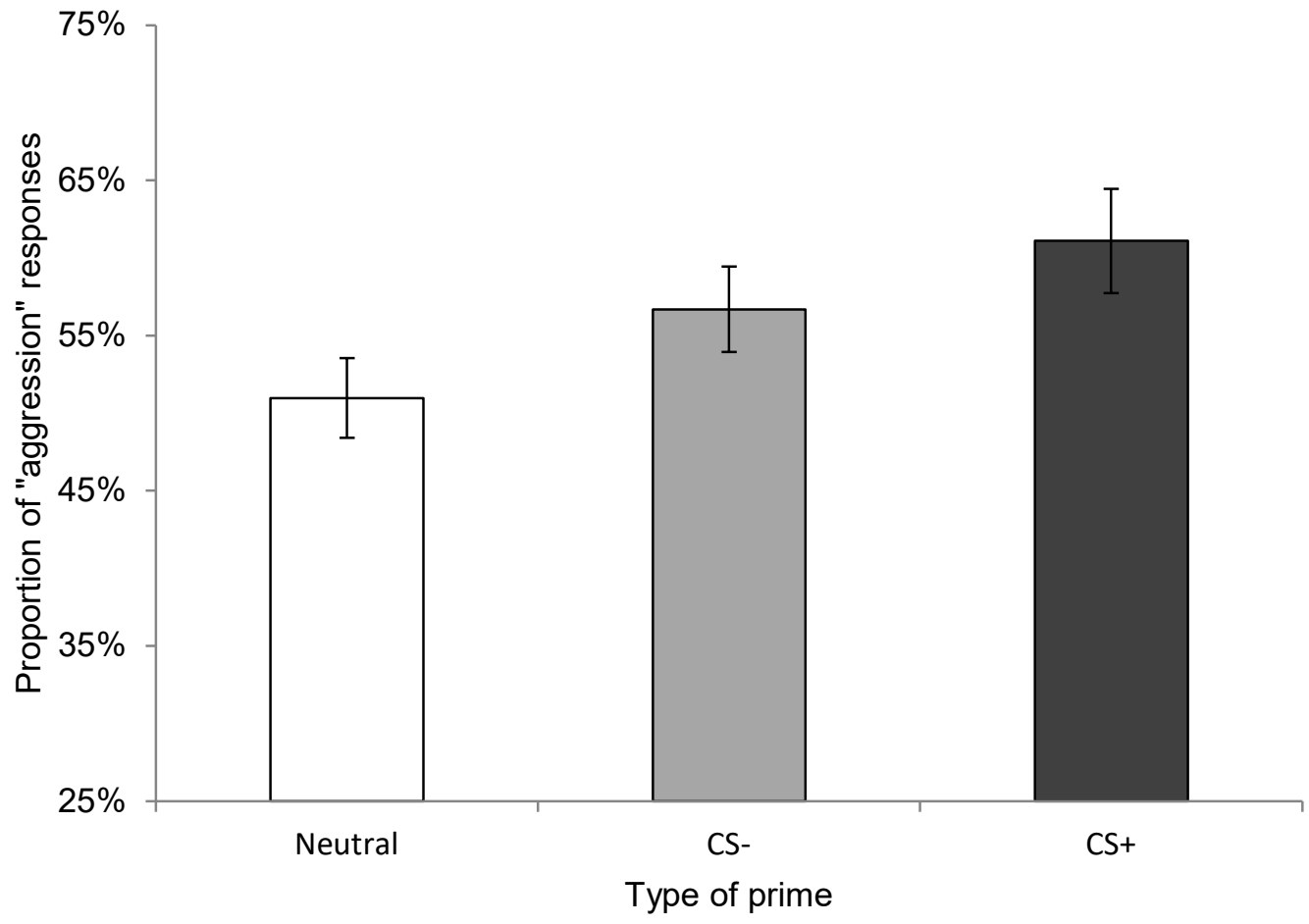

